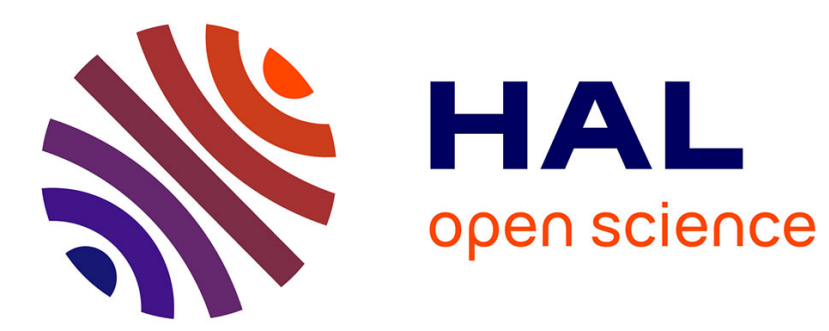

\title{
Vieillissement, Durabilité et Dégradation de Matériaux Composites Soumis à des Environnements Agressifs \\ Marco Gigliotti
}

\section{To cite this version:}

Marco Gigliotti. Vieillissement, Durabilité et Dégradation de Matériaux Composites Soumis à des Environnements Agressifs. 17èmes Journées Nationales sur les Composites (JNC17), Jun 2011, PoitiersFuturoscope, France. pp.16. hal-00597882

\section{HAL Id: hal-00597882 https://hal.science/hal-00597882}

Submitted on 2 Jun 2011

HAL is a multi-disciplinary open access archive for the deposit and dissemination of scientific research documents, whether they are published or not. The documents may come from teaching and research institutions in France or abroad, or from public or private research centers.
L'archive ouverte pluridisciplinaire HAL, est destinée au dépôt et à la diffusion de documents scientifiques de niveau recherche, publiés ou non, émanant des établissements d'enseignement et de recherche français ou étrangers, des laboratoires publics ou privés. 


\title{
Vieillissement, Durabilité et Dégradation de Matériaux Composites Soumis à des Environnements Agressifs
}

\section{Ageing, Durability and Degradation of Composite Materials Subjected to Aggressive Environment}

\author{
Marco Gigliotti ${ }^{1}$, \\ Laureat du Prix Daniel Valentin 2010 \\ 1: Institut Pprime - CNRS - ENSMA - Université de Poitiers - UPR 3346 \\ Département Physique et Mécanique des Matériaux \\ ENSMA - Téléport 2 - 1, Avenue Clément Ader - BP 40109 \\ F86961 FUTUROSCOPE CHASSENEUIL Cedex \\ e-mail : marco.gigliotti@ensma.fr,
}

\begin{abstract}
Résumé
L'AMAC a attribué le Prix Daniel Valentin 2010 à l'auteur pour son travail de recherche dans le domaine du vieillissement et de la durabilité de matériaux composites à matrice organique (CMO) soumis à des environnements agressifs et concernant, en particulier :

- le couplage hygro - thermo - mécanique lié à des variations cycliques de température et humidité [1 - 3] et le développement d'une technique expérimentale originale - employant des plaques composites asymétriques de type 0/90 - pour le suivi et la caractérisation de ces phénomènes [6 - 9],

- le couplage mécano - diffusif lié aux mécanismes de thermo oxydation à haute température et à haute pression [4 - 5], et la caractérisation des effets de ce couplage à différentes échelles,

- le couplage électro - mécanique ayant lieu dans des matériaux composites soumis à des courant électrique d'intensité intermédiaire [10] et l'effet de ce couplage sur la durée de vie des CMO.

Dans cet articles une partie de ces travaux est synthétiquement résumée, les problématiques majeures liées au vieillissement des CMO sont mises en relief, les principaux verrous à lever sont présentés et des pistes pour des futurs travaux de recherche dans ce domaine sont identifiées.
\end{abstract}

\begin{abstract}
AMAC gave the Daniel Valentin 2010 Award for his research work within the framework of ageing and durability of composite materials subjected to aggressive environments and concerning mainly:

- $\quad$ the hygro - thermo - mechanical coupling related to transient and cyclical hygrothermal fields [1 - 3] and the development of an original experimental technique employing 0/90 unsymmetric composite plates for the monitoring and the characterisation of such phenomena [6 - 9],

- the reaction - diffusion - mechanical coupling related to the thermo-oxidation phenomena occurring at high temperature and high pressure [4-5] and the characterisation of such coupling at different scales,

- the electro - mechanical coupling occurring in composite materials subjected to electrical currents of intermediate intensity [10] and the effect of such coupling on the durability of such materials.

The present paper reviews some of the cited work : the main issues concerning ageing in composite materials are discussed and perspectives for future studies in this research domain are identified.
\end{abstract}

Mots Clés : CMO, Vieillissement, Durabilité, Humidité, Température, Couplage électro - mécanique. Keywords : Composite Materials, Durability, Ageing, Temperature, Electro - Mechanical Coupling.

\section{Introduction}

L'emploi de matériaux composites à matrice organique $(\mathrm{CMO})$ pour la réalisation de structures aéronautiques permet un gain de masse entre $25 \%$ et $30 \%$ par rapport à des solutions plus classiques (alliages d'aluminium ou de titane) ; cependant, aujourd'hui, la masse de structures composites ne représente que $20 \%$ environ de la masse totale. En effet, ces matériaux ont de très bonnes propriétés spécifiques, résistance et rigidité, et une exceptionnelle résistance en fatigue dans des conditions 
environnementales 'standard', mais leurs performances se dégradent en présence d'environnements agressifs, suite à l'apparition de phénomènes de vieillissement.

Pour ces raisons, l'intégration des $\mathrm{CMO}$ dans des structures confrontées à des environnements agressifs demeure difficile [11] car des problèmes de durabilité se manifestent [11 - 14].

Le vieillissement des CMO résulte de l'action combinée de la température, de l'humidité, et de la pression de l'environnement mais aussi des conditions de leur mise en œuvre (type de procédé, cycle de cuisson induisant des contraintes résiduelles) et de la structure du matériau [12]; il correspond à une évolution souvent irréversible des propriétés du matériau et son étude nécessite des approches complexes, multi - physiques et multi - échelles.

La définition de durabilité est elle-même complexe : selon [12], la durée de vie est «le temps pendant lequel le matériau, ou plus précisément une de ses propriétés, va conserver sa fonctionnalité dans des conditions bien définies ». Cette définition doit être «interprétée » en fonction du type et de la nature du vieillissement : en outre, l'identification claire des paramètres dimensionnant et des chargements environnementaux affectant les mécanismes de vieillissement (et leur localisation) est souvent difficile [12].

Les difficultés liées à la caractérisation expérimentale des phénomènes de vieillissement (type d'essai, type de conditionnement, type d'éprouvette), à la détermination de conditions d'accélération de ces essais et à la «mesure» des durées de vie des CMO contribuent ultérieurement à complexifier les travaux de recherche dans ce domaine.

Les organismes de certification européens (JAA) ou américains (FAA) ont imposé des normes pour le dimensionnement des structures en CMO permettant de prendre en compte les conditions environnementales de service et fixant les conditions d'accélération des essais de vieillissement (JAR 25 - 603 [12]) : la plupart de ces normes sont "pragmatiques", basées sur le retour d'expérience, et il est clair qu'une compréhension fine des mécanismes ayant lieu pendant les processus de vieillissement est nécessaire pour pouvoir les élargir et les compléter.

L'exemple du vieillissement humide est très significatif, l'absorption d'eau, souvent réversible, induisant une baisse des propriétés mécaniques des CMO. Partant du principe qu'un CMO exposé à un environnement humide atteint souvent un état de saturation et que la baisse de ses propriétés peut être assez bien caractérisée, les normes suggèrent de prendre en compte directement dans le dimensionnement la chute des propriétés à saturation [11]. Cette procédure ignore ouvertement les phases transitoires (ou cycliques) du conditionnement et les mécanismes de dégradation qui ont lieu pendant ces phases, en particulier le développement de gradients de contraintes et de propriétés normalement absents dans un état dit «saturé »- qui peuvent interagir de façon complexe avec les sollicitations mécaniques.

Pour donner un autre exemple, les normes semblent être très vigilantes par rapport a la tenue à la foudre des structures de fuselage en $\mathrm{CMO}$ et préconisent l'utilisation de protections particulières (ex : grillage de bronze) contre le foudroiement [11]. Encore une fois cette façon de faire - qui concerne un type de phénomène assez particulier et assez rare - ne prend pas en compte les formes de vieillissement électrique ayant lieu dans des CMO soumis à la circulation de courants électriques parasites - même d'intensité relativement faible - pendant des durées très longues.

Les pièces structurales du futur avion supersonique (envisagé pour l'horizon 2020 - 2030) seront soumises à des sollicitations hygro - thermo - mécaniques cycliques assez importantes $[14,15]$ : les phénomènes de désorption d'humidité à hautes températures et les successifs passages à basses températures peuvent favoriser l'amorçage et la propagation de l'endommagement [1-3]. Dans les premiers stades de turboréacteurs (structures 'tièdes'), les températures en jeu sont de l'ordre de $120^{\circ} \mathrm{C}-190^{\circ} \mathrm{C}$ et la présence de l'oxygène dans le milieu environnant induit des processus de thermo-oxydation des matrices organiques conduisant à de sévères phénomènes de dégradation et une diminution significative de la durée de vie [4-5]. La réalisation de panneaux de fuselage en CMO (pour le futur A350 par exemple) va nécessiter une meilleure compréhension du comportement thermo - électro - mécanique couplé de ces matériaux dans une gamme de courants électriques intermédiaires - courants directs et alternatifs jusqu'à 10A, 900Hz [10]. La maitrise des phénomènes de dégradation associés à ces conditions d'utilisation nécessite la mise en place 
d'approches multi-physiques et multi-échelles, à la fois expérimentales et théoriques, et soulève des questions scientifiques, outre que technologiques, de grande ampleur et complexité.

L'AMAC a attribué le Prix Daniel Valentin 2010 à l'auteur pour son travail de recherche concernant :

- le couplage hygro - thermo - mécanique lié à des variations cycliques de température et humidité [1 - 3] et le développement d'une technique expérimentale originale - employant des plaques composites asymétriques de type 0/90 - pour le suivi et la caractérisation de ces phénomènes [6 - 9],

- le couplage mécano - diffusif lié aux mécanismes de thermo oxydation à haute température et à haute pression [4 - 5], et la caractérisation des effets de ce couplage à différentes échelles,

- le couplage électro - mécanique ayant lieu dans des matériaux composites soumis à des courants électriques d'intensité intermédiaire [10] et l'effet de ce couplage sur la durée de vie des CMO.

Dans cet article une partie de ces travaux [1 - 3, 6 - 9] est synthétiquement résumée, les problématiques majeures liées au vieillissement des CMO sont mises en relief, les principaux verrous à lever sont présentés et des pistes pour des futurs travaux de recherche dans ce domaine sont identifiées.

\section{Couplage hygro - thermo - mécanique lié à des variations cycliques de température et humidité.}

Ce chapitre présente synthétiquement le travail effectué par l'auteur dans le cadre du Programme de Recherche RRIT Supersonique 2001 concernant le couplage hygro - thermo - mécanique lié à des variations cycliques de température et humidité [1 - 3] et le développement d'une technique expérimentale originale - employant des plaques composites asymétriques de type 0/90 - pour le suivi et la caractérisation de ces phénomènes [6 - 9]. La section 2.1 présente les généralités sur la modélisation des contraintes internes hygrothermoélastiques, la section 2.2 les simulations des contraintes internes issues de ce modèle et le détail de leur mesure à travers l'emploi de plaques 0/90 asymétriques. La présence d'un endommagement du aux différentiels de température, au conditionnement humide et au cyclage thermique a été souligné dans la littérature [14]. La figure 1 montre une plaque stratifiée endommagée par un conditionnement cyclique, sans aucun chargement mécanique appliqué [15]. Il est clair aujourd'hui qu'un chargement hygrothermique peut provoquer des dommages sous la forme de microfissures dans la matrice, décollement fibre matrice, micro délaminage.

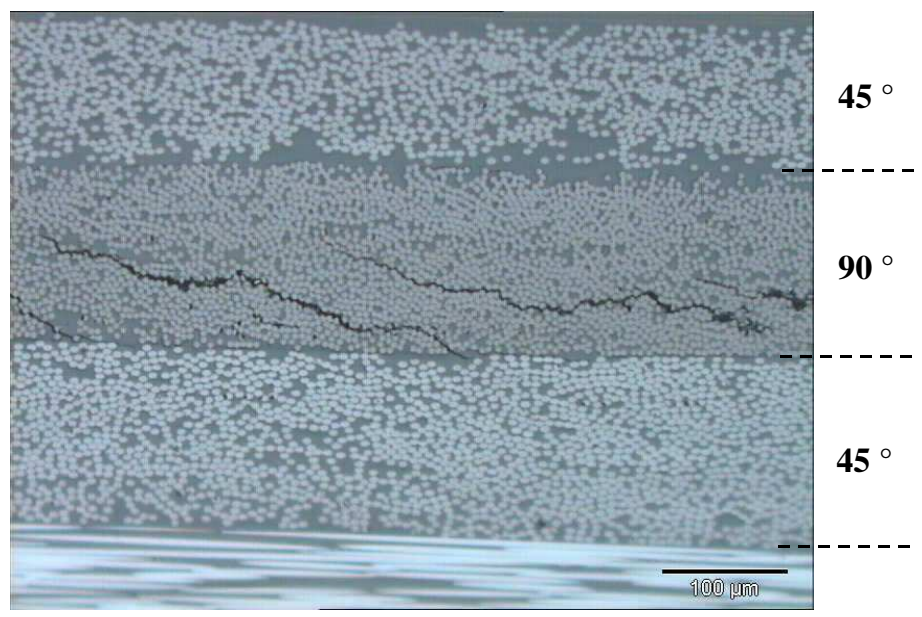

Fig. 1. Evidence d'endommagement dans des plaques composites soumises à un cyclage hygrothermique [15]. 


\subsection{Généralités sur la modélisation des contraintes internes hygrothermoélastiques cycliques}

Un problème hygrothermoélastique peut être modélisé en utilisant une théorie découplée, où la température, la concentration de l'eau diffusante (ou son potentiel chimique) et la déformation (ou la contrainte), c'est à dire les trois variables d'état du problème considéré, sont gouvernées par des équations indépendantes et peuvent être résolues séparément. Les équations découplées de l'hygrothermoélasticité peuvent en principe être déduites de celles de la théorie couplée ; pour cela, le flux diffusif doit être indépendant des contraintes. Le modèle de Fick suppose que le taux de transfert de la substance diffusante par unité de surface de section du solvant est proportionnel au gradient - normale à la section - de la concentration. Le coefficient de diffusion dépend seulement de la température. En supposant que la vitesse totale du système (polymère et milieu diffusant) est égale à zéro (en fait, elle peut être négligée), la conservation de la masse est exprimée par :

$$
\frac{\partial \mathrm{c}}{\partial \mathrm{t}}-\mathbf{D} \cdot \nabla \cdot \nabla \mathrm{c}=0
$$

où c est la concentration en eau, $\mathbf{D}=\mathbf{D}(\mathrm{T})=\mathrm{D}(\mathrm{T}) \mathbf{I}$ est le tenseur des coefficients de diffusion, dépendant de la température. La résolution de l'Eq. (1) avec les conditions initiales et aux limites appropriées, donne la concentration en eau en fonction du temps et de l'espace. Là loi de Fick peut être employée dans de nombreux cas pratiques, notamment, lorsque les relevés expérimentaux révèlent une indépendance entre le coefficient de diffusion et la concentration en eau. La concentration sur les surfaces, $\mathrm{c}_{\infty}$, peut être considérée constante : elle est habituellement liée à l'humidité relative externe par le biais d'une expression semi-empirique du type :

$$
\mathrm{c}_{\infty}=\mathrm{C}(\mathrm{RH})^{\mathrm{b}}
$$

où $\mathrm{C}$ et $\mathrm{b}$ sont des constantes dépendant du matériau et $\mathrm{RH}$ est l'humidité relative externe (\%). La dépendance du coefficient de diffusion en fonction de la température est représentée par une loi d'Arrhenius :

$$
\mathrm{D}=\mathrm{D}_{0} \mathrm{e}^{\left(-\frac{\Delta \mathrm{H}}{\mathrm{RT}}\right)}=\mathrm{Ae}^{\left(\frac{\mathrm{B}}{\mathrm{T}}\right)}
$$

où $\mathrm{D}_{0}$ est une constante dépendant du matériau, $\Delta \mathrm{H}$ est l'énergie d'activation et $\mathrm{R}$ est la constante des gaz parfaits. Finalement, A et $\mathrm{B}$ représentent deux paramètres à identifier. Les conditions d'essais permettant d'identifier les paramètres de la diffusion sont rarement représentatives des conditions « réelles » pour lesquelles humidité et température varient dans le temps. En fait, pour de nombreuses applications industrielles les conditions environnementales présentent un caractère périodique, les champs de concentration et de température en surface du solide sont des fonctions périodiques de période $\tau$. Dans ce cas, les processus de diffusion se révèlent plus complexes à modéliser. Les variations de température et d'humidité ont des répercussions sur le coefficient de diffusion, qui dépend de la température, et sur les conditions aux limites, qui dépendent de l'humidité relative. Les fluctuations de l'environnement externe induisent des fluctuations à l'intérieur du solide seulement dans une zone proche des surfaces latérales, dont l'étendue peut être déterminée explicitement, et vaut [3] :

$$
\mathrm{e}_{0}=2 \sqrt{\pi \int_{0}^{\tau} \mathrm{D}(\mathrm{t}) \mathrm{dt}}=2 \sqrt{\pi \Delta(\tau) \tau}
$$


$\mathrm{e}_{0}$ prend en compte les variations périodiques du coefficient de diffusion $\mathrm{D}$ sur un cycle. $\Delta(\tau)$ est le coefficient de diffusion moyen sur une période. Il doit être noté que $\mathrm{e}_{0}$ dépend à la fois des propriétés du matériau, à travers le coefficient de diffusion, mais aussi du cycle hygrothermique par le biais de la période $\tau$. Finalement, $\mathrm{e}_{0}$ est l'épaisseur d'une région frontière dans laquelle un régime fluctuant, directement affecté par les conditions environnementales cycliques, prévaut. Une solution analytique approchée pour le problème hygrothermique cyclique peut être explicitée en introduisant des quantités moyennes [1 - 3]. Pour une plaque de faible épaisseur le champ de température peut être considéré uniforme et la diffusion d'eau essentiellement uni-dimensionnelle : de plus, si les plis sont constitués du même matériau, la plaque est considérée homogène vis à vis du processus de diffusion. Le problème fluctuant peut être réduit en un problème équivalent avec des concentrations aux limites et un coefficient de diffusion constants : plus précisément, en introduisant les grandeurs moyennes telles que le coefficient de diffusion moyen, $\Delta(\tau)$, et les concentrations moyennes en surface $\hat{\mathrm{c}}_{\infty}$ :

$$
\Delta(\tau)=\int_{0}^{\tau} \mathrm{D}(\mathrm{t}) \mathrm{dt}, \hat{\mathrm{c}}_{\infty}=\frac{1}{\Delta(\tau)} \int_{0}^{\tau} \mathrm{D}(\mathrm{t}) \mathrm{c}_{\infty}(\mathrm{t}) \mathrm{dt}
$$

la concentration à l'intérieur de la plaque est de la forme (concentration initiale nulle) :

$$
\begin{gathered}
\mathrm{c}(\mathrm{z}, \mathrm{N}-\mathrm{k})=\hat{\mathrm{c}}_{\infty}-\frac{4 \hat{\mathrm{c}}_{\infty} \pi}{\beta^{2}} \sum_{\mathrm{n}=0}^{\infty} \sum_{\mathrm{i}=0}^{\mathrm{k}-1}\left((-1)^{\mathrm{n}}(2 \mathrm{n}+1) \exp \left(-\frac{(2 \mathrm{n}+1)^{2} \pi^{2}(\mathrm{~N}-\mathrm{i})}{\beta^{2}}\right)\right) \\
\cos \left(\frac{(2 \mathrm{n}+1) \pi \mathrm{Z}}{\mathrm{e}}\right) \\
\forall(-\mathrm{e} / 2)<\mathrm{z}<(\mathrm{e} / 2)
\end{gathered}
$$

où e représente l'épaisseur de la plaque et :

$$
\beta=\sqrt{\frac{\mathrm{e}^{2}}{\Delta(\tau)}}
$$

$\mathrm{N}$ est le nombre de cycles pour atteindre la saturation et l'Eq. 6 fournit la concentration à l'intérieur de la plaque après $(\mathrm{N}-\mathrm{k})$ cycles. Finalement, le champ de concentration complet est composé de la solution analytique Eq. (6) - valable seulement à l'intérieur de la plaque - et d'une solution fluctuante aux voisinages des surfaces obtenue par un calcul aux différences finies. Les contraintes internes hygrothermoélastiques peuvent être calculées à partir des champs hygrothermiques en utilisant les équations d'équilibre et la loi constitutive Eq. (8), qui prend en compte un comportement élastique linéaire. Le tenseur de rigidité $\mathbb{C}$ dépend de la concentration et de la température dans le cas général :

$$
\mathbf{S}=\mathbb{C}(\mathrm{c}, \mathrm{T}):\left(\mathbf{E}-\mathbf{E}^{\mathrm{HT}}\right)
$$

où $\mathbf{S}$ est le tenseur des contraintes et $\mathbf{E}$ est le tenseur des déformations totales. Les déformations libres d'origine hygrothermique, $\mathbf{E}^{\mathrm{HT}}$, sont exprimées par les relations suivantes :

$$
\mathbf{E}^{\mathrm{HT}}=\alpha \Delta \mathrm{T}+\beta \Delta \mathrm{m}
$$

ou $\boldsymbol{\alpha}$ et $\boldsymbol{\beta}$ sont les tenseurs des dilatations thermique et hygroscopique, tandis que $\Delta \mathrm{T}$ et $\Delta \mathrm{m}$ représentent les incréments de température et de teneur en eau $\left(\mathrm{m}=\mathrm{c} / \rho_{\mathrm{s}}, \rho_{\mathrm{s}}\right.$ étant la masse 
volumique du polymère). La solution d'un problème hygrothermoélastique dans le cadre de la théorie classique des plaques stratifiées implique l'utilisation de champs hygrothermiques approchés par des fonctions linéaires par morceaux [3].

Des simulations des champs de concentration et des contraintes hygrothermoelastiques effectuées en utilisant différents modèles structuraux (cylindres épais, plaques épaisses 3D, plaques minces) montrent que [1] :

- les conditions transitoires/cycliques dans des cylindres homogènes ou composites UD peuvent être simulées en utilisant la théorie classique des plaques quelle que soit la valeur du rapport entre le rayon interne et l'épaisseur du cylindre $\left(\mathrm{R}_{\mathrm{int}} / \mathrm{e}\right)$,

- dans des matériaux multicouches la convergence de la solution analytique pour les cylindres vers la solution approchée pour les plaques minces est très lente : l'hétérogénéité joue un rôle fondamental sur les différences observées entre les deux approches,

- la solution analytique pour les plaques 3D converge vers la solution des plaques minces pour des matériaux homogènes ou multicouches,

- le modèle approché (plaque mince) donne une excellente approximation du résultat exact même dans le cas de profils de concentration non uniformes, c'est à dire, caractérisés par des gradients de concentration importants.

2.2. Simulations des contraintes internes hygrothermoélastiques cycliques induites par des conditions environnementales typiques d'un vol supersonique et leur mesure expérimentale

Le modèle hygrothermoélastique approché (plaques minces), présenté dans le chapitre précédent, est utilisé pour simuler les contraintes internes induites par des conditions environnementales typiques d'un vol supersonique. Le cycle hygrothermique dit supersonique est détaillé dans la figure 2 [3]. Dans ce cycle quatre points sont considérés :

- point $\mathrm{A}$, qui correspond à des conditions environnementales au sol,

- point $\mathrm{B}$, à $-55^{\circ} \mathrm{C}$ et $0 \% \mathrm{HR}$, qui correspond à la phase subsonique du vol,

- point $\mathrm{C}$, à $130^{\circ} \mathrm{C}$ et $0 \% \mathrm{HR}$, qui correspond à la phase supersonique du vol,

- point $\mathrm{D}$, qui correspond a la fin du vol.

Un conditionnement initial à $23^{\circ} \mathrm{C}$ et $50 \% \mathrm{HR}$ pendant 3 mois est inclus dans les calculs pour simuler la phase de manutention au sol qui aboutit au point A. Cet état est noté comme l'état initial humide. Les simulations sont effectuées pour une plaque composite carbone-époxyde IM7/977-2 $\left[0_{4} / 90_{4}\right]_{S}$ d'épaisseur totale $4 \mathrm{~mm}$.

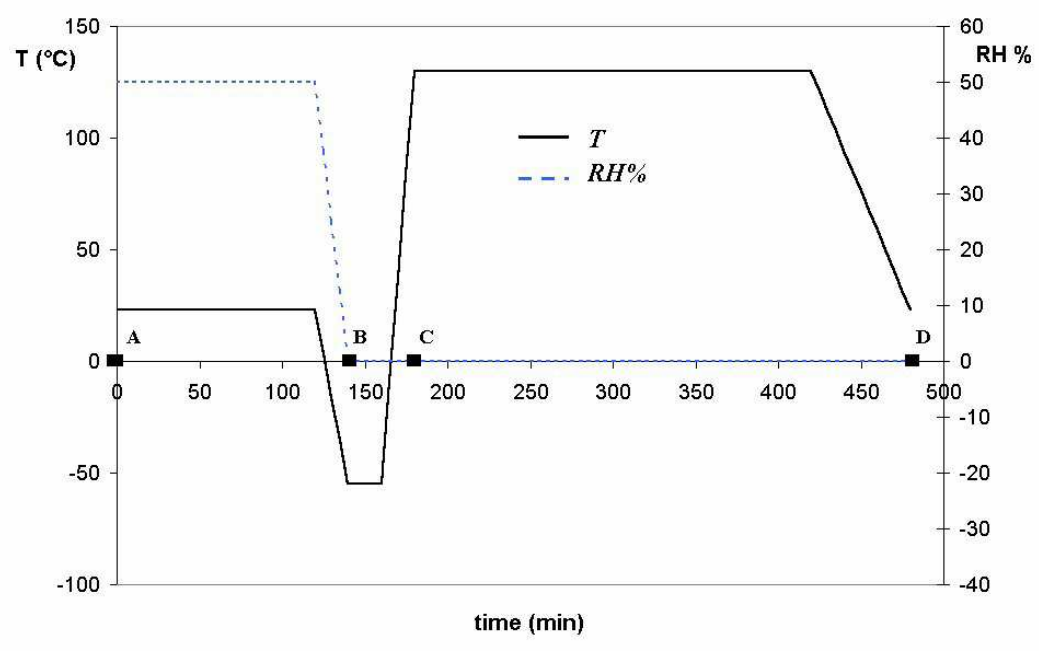

Fig. 2. Description détaillée du cycle hygrothermique supersonique [3].

Dans la figure 3 [3], les champs de concentration en eau au cours des cycles 1 et 50 sont présentés. Nous vérifions que les solutions analytiques et numériques au cœur de la plaque sont identiques. 
Les simulations montrent que l'étendue de la zone fluctuante, $\mathrm{e}_{0}$, est, pour ce type de cycle et pour ce matériau, égale à $0.5 \mathrm{~mm}$, ce qui est également prédit par 1Eq. (4).
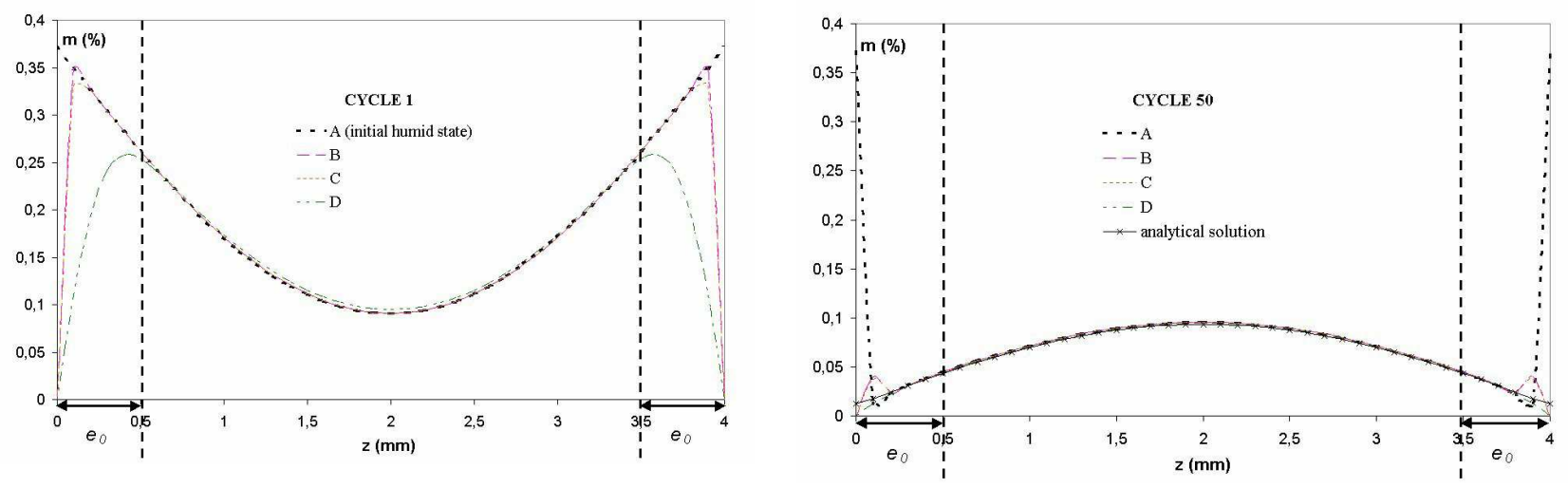

Fig. 3. Champs hygroscopiques au cours des cycles 1 et 50 [3].

L'effet moyen du cyclage supersonique consiste en un séchage progressif de la plaque, qui se trouve dans un état quasi-sec après 300 cycles environ. La figure 4 [3] présente les contraintes $S_{x x}$ et $S_{y y}$ aux points A, B, C et D du cycle 1. La figure 5 [3] montre l'évolution des contraintes internes $S_{\mathrm{yy}}$ tout au long du cyclage supersonique. Les contraintes résiduelles de fabrication sont inclues dans les simulations, en considérant un différentiel de température $\Delta \mathrm{T}=\left(\mathrm{T}_{\text {ambiante }}-\mathrm{T}_{\text {cuisson }}\right)=-187^{\circ} \mathrm{C}$.
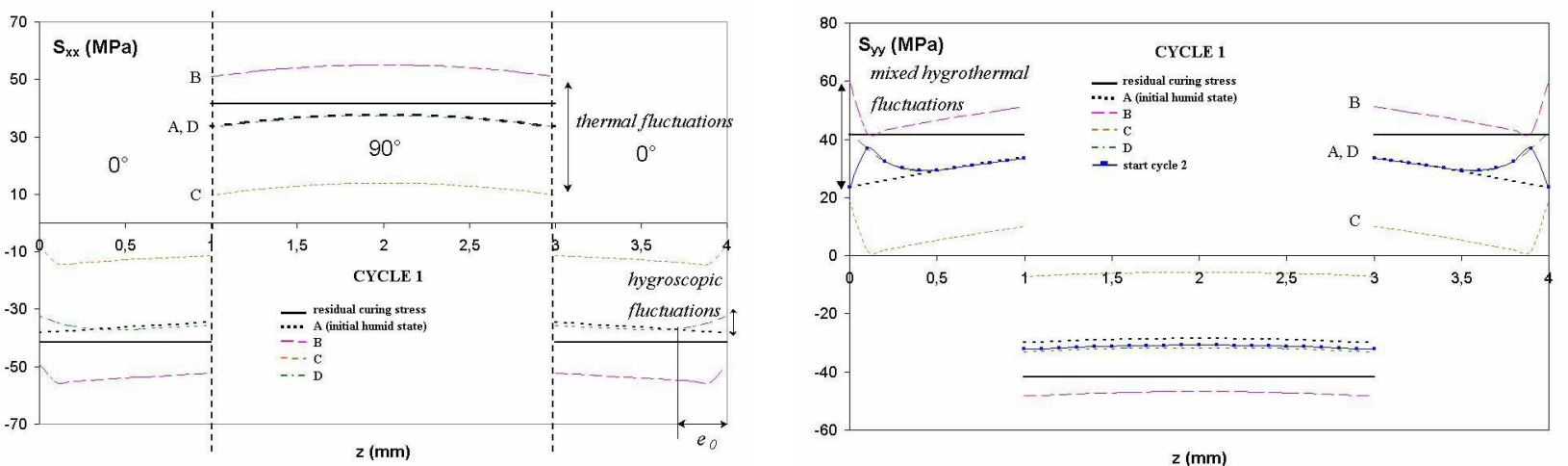

Fig. 4. Champs de contraintes internes hygrothermoélastiques $S_{x x}$ et $S_{y y}$ pendant le cycle 1 [3].

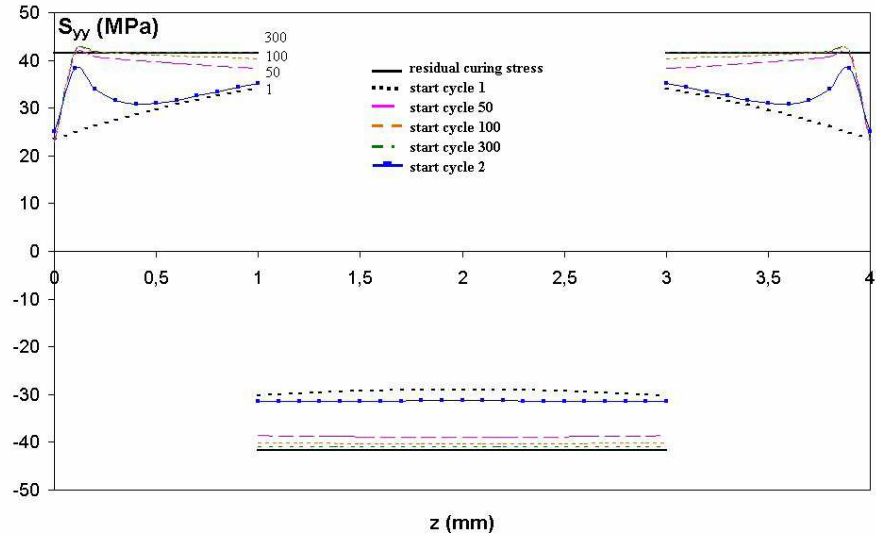

Fig. 5. Champs de contraintes internes hygrothermoélastiques $S_{y y}$ pendant le cyclage supersonique [3].

Les contraintes internes sont élevées après la fabrication. Les contraintes résiduelles de cuisson, qui sont représentées dans chaque figure comme référence, sont uniformes dans chaque pli et atteignent 
des valeurs de $40 \mathrm{MPa}$ environ. Lors du passage à $-55^{\circ} \mathrm{C}$ (point $\mathrm{B}$ ) les contraintes transverses aux fibres atteignent des valeurs positives d'environ $50 \mathrm{MPa}$, ce qui peut entraîner l'amorçage de l'endommagement dans le matériau. Les fluctuations thermiques provoquent des variations de contraintes internes sur l'ensemble de la plaque : les fluctuations de nature hygroscopique peuvent être appréciées lors du passage de la fin d'un cycle au début du cycle successif et provoquent des variations de contraintes dans une zone de la plaque proche des surfaces latérales, d'épaisseur égale à $\mathrm{e}_{0}$. Le séchage dû aux cycles de service produit une réapparition progressive des contraintes résiduelles de fabrication: l'amplitude des fluctuations augmente avec le nombre de cycles, par contre l'étendue de la zone fluctuante reste égale à $\mathrm{e}_{0}$.

Les Figs. 1-4 nous montrent qu'il est possible de simuler, avec une bonne approximation, l'état de contraintes internes engendré par des conditions environnementales complexes. Cette approche, même si elle peut être enrichie en tenant compte d'un comportement plus complexe (viscoélasticité, endommagement...), est extrêmement utile pour la conception et l'optimisation des structures. Toutefois, il est nécessaire de mesurer ces contraintes afin de valider les modèles, en particulier dans des conditions complexes, en présence de champs transitoires et cycliques.

Pour ce faire, l'utilisation de plaques asymétriques de type [0/90] est employée [6 - 9] : ces échantillons se déforment lorsqu'ils sont exposés aux environnements hygrothermiques et - à travers leur distorsion - sont capables de suivre des évolutions transitoires assez complexes. La technique optique de la projection de franges peut être utilisée pour suivre la distorsion de ces plaques en régime transitoire : il s'agit d'une technique sans contact, facile à calibrer et pour laquelle aucune hypothèse sur le champ de déplacement mesuré n'est nécessaire. En particulier, cette technique est capable de capturer les déplacements hors plan tout le long du champ bidimensionnel mesuré, sans hypothèses sur la déformée expérimentale. Afin d'exploiter ce type d'expérience, il est nécessaire de modéliser correctement le comportement des plaques composites asymétriques déformées sous l'effet des champs hygrothermiques. Pour cela, un modèle variationnel approché (méthode de Rayleigh-Ritz) géométriquement non-linéaire (petites déformations, rotations modérées) de plaque peut être employé, permettant d'obtenir de façon approchée les configurations d'équilibre des plaques déformées [6 - 9].

Pour déterminer les configurations d'équilibre, on part du principe que, à chaque instant du conditionnement transitoire ou cyclique, l'énergie potentielle totale $\mathrm{E}_{\mathrm{T}}$ admet un extremum local. L'équilibre est stable lorsque l'énergie potentielle totale admet un minimum :

$$
\left\{\begin{array}{l}
\delta \mathrm{E}_{\mathrm{T}}=\delta\left(\mathrm{E}_{\mathrm{D}}-\mathrm{L}\right)=0 \text { condition d'équilibre } \\
\delta^{2} \mathrm{E}_{\mathrm{T}}>0 \text { équilibre stable }
\end{array}\right.
$$

$\mathrm{E}_{\mathrm{D}}$ est l'énergie de déformation - prenant en compte les effets mécaniques, thermiques et hygroscopiques - tandis que L est le travail externe, c'est-à-dire, le travail du aux efforts externes appliqués. L'Eq. (10) peut être résolue en utilisant la méthode de Rayleigh-Ritz, de Galerkin ou des éléments finis.

Les Figs. 6 et 7 [8] montrent la capacité de la méthode expérimentale à capter les changements de forme ayant lieu lors d'un conditionnement hygrothermique complexe et à identifier un modèle numérique simulant cet état déformé.

La Fig. 8 montre la capacité de la méthode à suivre un conditionnement hygrothermique complexe, caractérisé par les cycles température - humidité du vol supersonique : dans la Fig. 8 l'évolution de la courbure adimensionnée, $a_{*}^{*}$, d'une plaque composite $0 / 90$ en fonction du temps de conditionnement adimensionné, $t^{*}$ est illustrée.

Il est intéressant de noter que la technique expérimentale est capable de caractériser toutes les phases - transitoires ou cycliques - du conditionnement hygroscopique et - en particulier - de mesurer séparément les fluctuations d'origine thermiques (points B - C) et celles d'origine hygroscopiques (points A - D). 


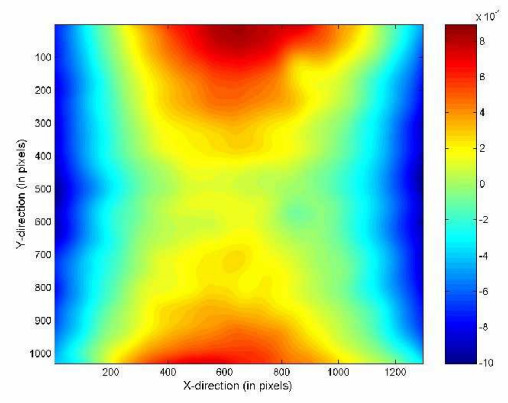

experimental shape

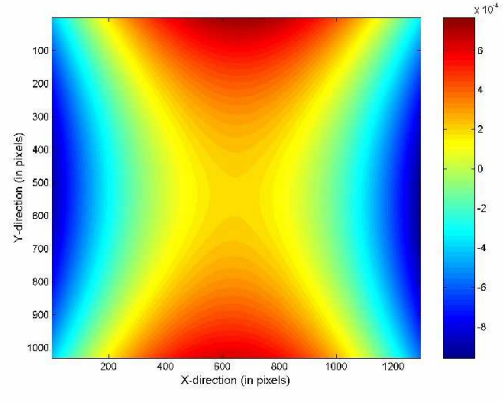

numerical fitting

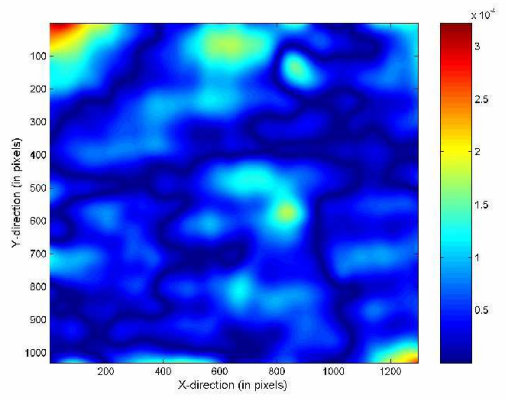

absolute error

Fig. 6. Mesure par projection de franges de la distorsion d'une plaque 0/90 asymétrique au cours du conditionnement hygrothermique transitoire (forme selle de cheval) [8].
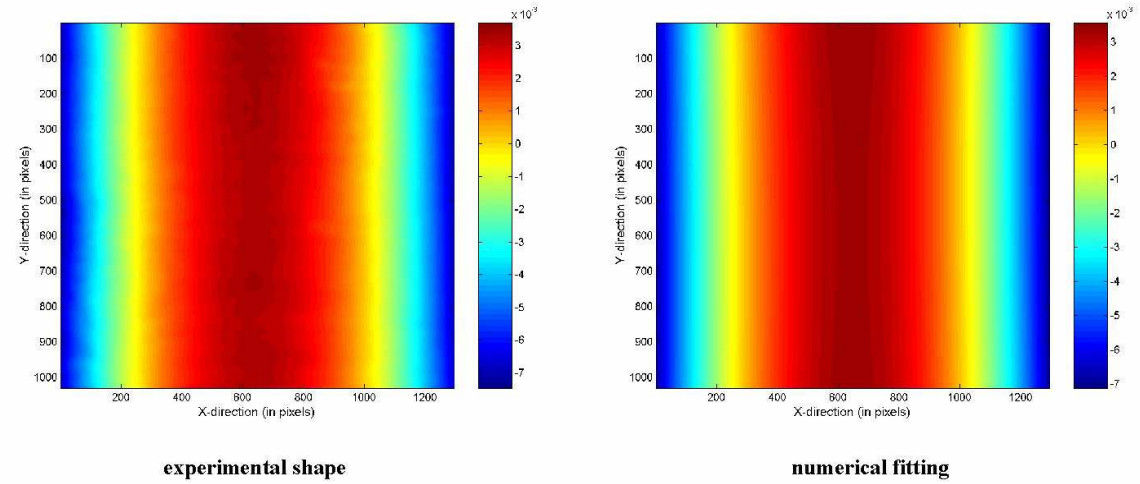

numerical fitting

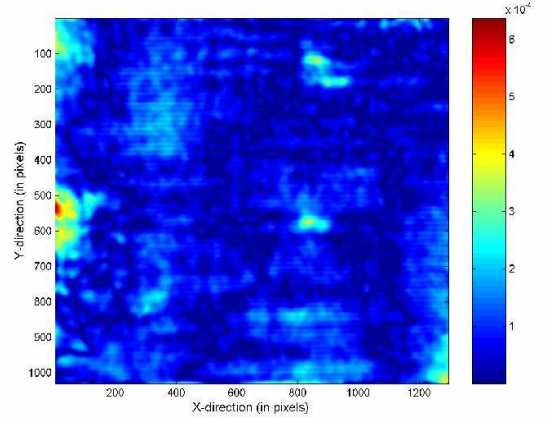

absolute error

Fig. 7. Mesure par projection de franges de la distorsion d'une plaque 0/90 asymétrique au cours du conditionnement hygrothermique transitoire (forme cylindrique) [8]. 


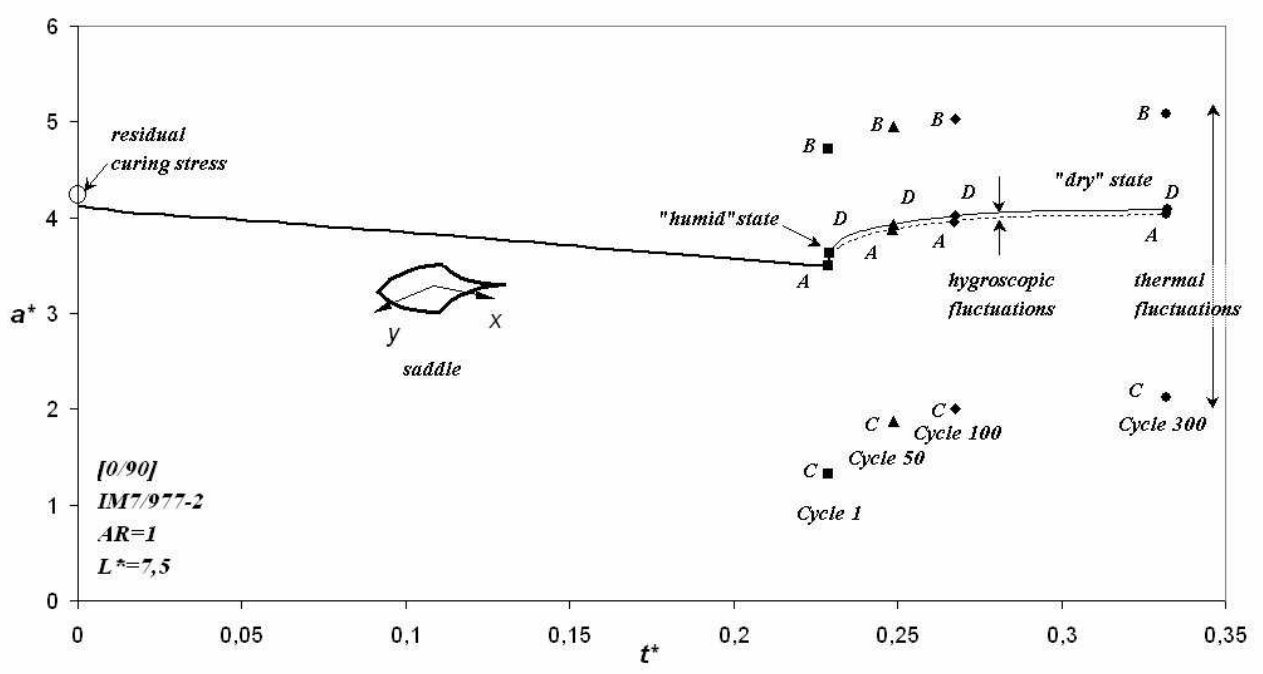

Fig. 8. Evolution de la courbure d'une plaque asymétrique 0/90 pendant le conditionnement hygrothermique du vol supersonique.

Le dialogue entre la méthode variationnelle numérique et la technique expérimentale permet d'identifier des configurations structurales de plaques 0/90 optimisées, pour lesquelles des techniques expérimentales plus simples que la projection de frange et des modèles de calcul simplifiés (théorie linéaire classique des plaques composites) peuvent être employés. C'est le cas de plaques 0/90 rectangulaires caractérisées par des valeurs élevées du rapport longueur/largeur.

\section{Conclusions}

Cet article résume synthétiquement une partie des travaux de recherche de l'auteur concernant le couplage hygro - thermo - mécanique lié à des variations cycliques de température et humidité [1 3] et le développement d'une technique expérimentale originale - employant des plaques composites asymétriques de type 0/90 - pour le suivi et la caractérisation de ces phénomènes [6 9]. Les résultats montrent qu'il est possible de simuler et de mesurer pertinemment les contraintes internes engendrées par des conditions environnementales complexes, dans des régimes transitoires et cycliques.

L'application de la technique des plaques 0/90 asymétriques à des conditions de vieillissement encore plus complexes (thermo oxydation) est en cours de développement [16 - 17]. Le développement de modèles prenant en compte un couplage fort entre champs hygro - thermo chimiques et les champs mécaniques est également en cours [4].

Il est clair qu'une compréhension fine des mécanismes ayant lieu pendant les processus de vieillissement passe par la mise en place d'approches multi-physiques et multi-échelles, à la fois expérimentales et théoriques, qui soient capable de relever les mécanismes de base impliqués dans ces processus. C'est l'objet des futurs travaux de recherche.

\section{Remerciements.}

Je souhaite remercier l'AMAC, pour m'avoir attribué le prix Daniel Valentin 2010, Marie Christine Lafarie-Frenot et Jean-Claude Grandidier (Institut Pprime - ENSMA Poitiers), pour m'avoir accueilli au sein de leur équipe de recherche. Je tiens à remercier également Alain Vautrin (EMSE Saint-Etienne) et Michael Wisnom (University of Bristol). Un remerciement particulier va à ma femme, Emanuela, pour son amour et pour son soutien pendant ces années.

\section{Références}

[1] Gigliotti, M., Jacquemin, F., Vautrin, A. « Assessment of Approximate Models to Evaluate Transient and Cyclical Hygrothermoelastic Stress in Composite Plates ». International Journal of Solids and Structures, Vol. 44, pp. 733-759, 2007. 
[2] Gigliotti, M., Jacquemin, F., Molimard, J., Vautrin A. « Modelling and Experimental Characterisation of Hygrothermoelastic Stress in Polymer Matrix Composites ». Macromolecular Symposia, Vol. 247, pp. 199-210, 2007.

[3] Gigliotti, M., Jacquemin, F., Vautrin, A. «Internal Stresses in Composite Laminates due to Cyclical Hygrothermal Loading. Application to Supersonic Flight». AIAA Journal, Vol. 42, pp. 2600-2605, 2004.

[4] Gigliotti, M., Grandidier, J.C. «Chemo-Mechanics Couplings in Polymer Matrix Materials Exposed to ThermoOxidative Environments ». Comptes Rendus Mécanique, Vol. 338, pp. 164-175, 2010.

[5] Gigliotti, M., Vu, D.Q., Olivier, L., Lafarie-Frenot, M.C., Grandidier, J.C. « Oxidation Induced Shrinkage and Stress in Composite Materials at High Temperatures: Numerical Simulations and Experimental Assessment ». ICCM 17, 17th International Conference on Composite Materials, July 29-July 31 2009, Edinburgh, UK.

[6] Gigliotti, M., Wisnom, M. R., Potter, K. D. « Loss of Bifurcation and Multiple Shapes of Thin [0/90] Unsymmetric Composite Plates Subject to Thermal Stress ». Composites Science and Technology, Vol. 64, pp. 109-128, 2004.

[7] Gigliotti, M., Jacquemin, F., Vautrin, A. « On the Maximum Curvatures of 0/90 Plates Under Thermal Stress ». Composite Structures, Vol. 68, pp. 177-184, 2005.

[8] Gigliotti, M., Molimard, J., Jacquemin, F., Vautrin, A. « On the Nonlinear Deformations of Thin Unsymmetric 0/90 Composite Plates Under Hygrothermal Loads ». Composites Part A: Applied Science and Manufacturing, Vol. 37, pp. 624-629, 2006.

[9] Gigliotti, M., Jacquemin, F., Molimard, J., Vautrin A. « Transient and Cyclical Hygrothermoelastic Stress in Laminated Composite Plates. Modelling and Experimental Assessment ». Mechanics of Materials, Vol. 39, pp. 729-745, 2007.

[10] Gigliotti, M., Grandidier, J.C., Lafarie-Frenot, M.C., Marchand, D. « Development of Experimental and Modelling Tools for Electro - Mechanical Fatigue Tests in Composite Materials for Aircraft Applications ». ECCM14, 7-10 June 2010, Budapest, Hungary.

[11] Cinquin, J. «Les Composites en Aerospatiale ». Techniques de l'Ingénieur, AM 5645.

[12] Mortaigne, B. «Vieillissement des Composites - Mécanismes et Méthodologie d'Etude ». Techniques de l’Ingénieur, AM 5320.

[13] Mortaigne, B. «Vieillissement des Composites - Evolution des Propriétés et Modélisation ». Techniques de l'Ingénieur, COR 113.

[14] Gates, T.S. « Durability Assessment of Polymeric Composites for High Speed Civil Transport ». Proceedings of DURACOSYS 99, Bruxelles, Belgique, July 11-14, 1999.

[15] Jedidi-Bouden, J. «Contribution à la caractérisation en cyclage hygrothermique d'un matériau composite. Application à l'avion supersonique » Thèse de Doctorat, Ecole des Mines de Saint-Etienne, 2005.

[16] Gigliotti, M., Grandidier, J.C., Lafarie-Frenot, M.C. «The Employment of 0/90 Unsymmetric Samples for the Characterisation of the Thermo-oxidation Behaviour of Composite Materials at High Temperatures ». Composite Structures, Vol. 93, pp. 2109-2119, 2011.

[17] Minervino, M., Gigliotti, M., Lafarie-Frenot, M.C., Grandidier, J.C. «L'Emploi de Plaques 0/90 Asymétriques Pour la Caractérisation des Phénomènes de Thermo-Oxydation dans les Matériaux Composites à Haute Température ». Proceedings of JNC17, Poitiers, France, June 15-17, 2011. 